\title{
SÍNTESIS DE NANOCOMPUESTOS POLIMÉRICOS CON GRAFENO Y SU CARACTERIZACIÓN MECÁNICA
}

\author{
Mercedes Puca Pacheco ${ }^{a^{*}}$, Enrique Tacuri Calanchi ${ }^{a}$, Agérico Pantoja Cadillo ${ }^{a}$, \\ María Guadalupe Neira Velázquez ${ }^{\mathrm{b}}$, Gonzalo Canché Escamilla ${ }^{\mathrm{c}}$.
}

\begin{abstract}
RESUMEN
Los nanocompuestos poliméricos con grafeno se obtuvieron por polimerización heterogénea en semicontinuo in situ. Sus propiedades mecánicas se determinaron en las películas formadas con diferentes concentraciones de grafeno. El proceso de obtención de los nanocompuestos consistió en dos etapas: En la primera etapa el grafeno fue sintetizado por el método de Hummer modificado. En la segunda etapa consistió en la dosificación de estireno (St), acrilato de butilo (AB) y metacrilato de alilo (ALMA); a un caudal de $0,5 \mathrm{~mL} / \mathrm{min}$, sobre la dispersión de grafeno obtenida en la primera etapa. Se realizó la caracterización de las películas poliméricas por medio de ensayos mecánicos, diámetros promedios de partícula mediante la técnica de dispersión dinámica de luz (DLS) y el análisis cualitativo de grupos funcionales por espectroscopia infrarroja.

De acuerdo con los resultados obtenidos, los látexes sintetizados tuvieron una conversión final superior al $87,2 \%$, y con un diámetro promedio de partícula comprendida entre 48,5 y $92,0 \mathrm{~nm}$, cuyos diámetros aumentaron a medida que aumentaba la concentración de grafeno. Se obtuvo un aumento en su módulo de Young y una disminución en el porcentaje de deformación a rotura a medida que aumentaba el contenido de grafeno en el nanocompuesto polimérico. El mayor módulo elástico fue 5,28 MPa para un contenido másico de grafeno de $2 \%$.
\end{abstract}

Palabras claves: Grafeno, nanocompuestos, propiedades mecánicas, películas poliméricas.

\section{SYNTHESIS OF POLYMERIC NANOCOMPOSITES WITH GRAPHENE AND ITS MECHANICAL CHARACTERIZATION}

\begin{abstract}
Polymeric nanocomposites with graphene were obtained by semicontinuous heterogeneous polymerization in situ. Its mechanical properties were determined in the films formed with

\footnotetext{
a Facultad de Química e Ingeniería Química, Universidad Nacional Mayor de San Marcos UNMSM.Av. Venezuela Cdra. 34, Lima 14, Perú. email: mpucap@hotmail.com

${ }^{\text {b }}$ Departamento de Síntesis de Polímeros, Centro de Investigación en Química Aplicada Blvd, Enrique Reyna Hermosillo, No. 140, col San José de los Cerritos, CP 25294, Saltillo, Coahuila, México CIQA. Saltillo - México.

c Unidad de Materiales, Centro de Investigación Científica de Yucatán A.C., Calle 43, No. 130, CP 97200, Mérida, Yucatán, México.
} 
different concentrations of graphene. The process of obtaining the nanocomposites consisted of two stages: In the first stage the graphene was synthesized by the modified Hummer method. The second stage consisted of the dosing of styrene (St), butyl acrylate (AB) and allyl methacrylate (ALMA); at a flow rate of $0.5 \mathrm{~mL} / \mathrm{min}$, on the graphene dispersion obtained in the first stage. The characterization of the polymer films were done by means of mechanical tests, average particle diameter by dynamic Light Scattering (DLS) technique, and the qualitative analysis of functional groups was carried out by infrared spectroscopy.

According to the results obtained, the latexes synthesized had a final conversion greater than $87.2 \%$, and with a particle average diameter between 48.5 and $92.0 \mathrm{~nm}$, whose diameters increased as the graphene concentration increased. An increase in its Young's modulus and decrease in the percentage of the deformation at break were obtained as the graphene content in the polymer nanocomposite was increased. The largest elastic modulus of 5.28 MPa for the nanocomposite containing $2 \%$ of graphene.

Key words: Graphene, nanocomposites, mechanical properties, polymer films.

\section{INTRODUCCIÓN}

Actualmente los nanocompuestos poliméricos están presentando un alto interés debido a que pueden mejorar las propiedades de los polímeros. Los copolímeros de estireno y acrilato de butilo generalmente son empleados en películas poliméricas, recubrimientos, mejoradores de impacto, entre otros. Al combinarse un material rígido como el poliestireno con un material ahulado como es el poliacrilato de butilo, es posible su empleo en películas poliméricas, pero generalmente estos materiales tienden a degradarse por cambios térmicos, y como consecuencia disminuir sus propiedades mecánicas.

Los nanocompuestos poliméricos son materiales que se caracterizan por la dispersión homogénea de partículas de dimensiones nanométricas (menores de $100 \mathrm{~nm}$ ) dentro de una matriz polimérica. En los materiales compuestos convencionales y, por tanto, con un componente, habitualmente inorgánico no nanoestructurado, existe una separación clara a nivel macroscópico entre las fases orgánica e inorgánica y donde el alto contenido de la carga conduce a una alta densidad, todo ello en detrimento de las propiedades y de la mejora del compuesto final. Por el contrario, los nanocompuestos poliméricos presentan un importante aumento de las características mecánicas y térmicas de las matrices que como acabamos de decir no se pueden conseguir a partir de los macrocompuestos. Un trabajo pionero sobre la preparación de nanocompuestos poliméricos fue llevado a cabo por Usuki y col. (1993), utilizando el sistema polímero-arcilla donde las cadenas del polímero (poliamida-6) se intercalaron entre los estratos de los silicatos ${ }^{1}$. Estos sistemas han tenido, posteriormente, gran aceptación debido a las sorprendentes propiedades mecánicas y térmicas respecto a los polímeros puros y a otros compuestos con cargas convencionales. Igualmente, otras nanopartículas están siendo muy utilizadas para obtener nanocompuestos poliméricos: nanocerámicas, nanopartículas metálicas, distintos materiales carbonosos (fullerenos, 
nanotubos de carbono, diferentes tipos de grafeno, nanodiamante), etc. De todos ellos, el grafeno ocupa un lugar destacado y está permitiendo abrir nuevas vías al desarrollo de materiales funcionales. Precisamente, sus propiedades excelentes y su obtención a partir de un material barato como es el grafito, es lo que explica el interés por este material y las altas prestaciones que se consiguen en distintos nanocompuestos poliméricos. Recientes estudios demuestran la alta conductividad térmica del grafeno ${ }^{2}$, la resistencia mecánica comparable a los nanotubos de carbono ${ }^{3}$, las buenas propiedades de transporte ${ }^{4}$, y su considerable efecto termoeléctrico ${ }^{5}$. Con el fin de explotar estas propiedades, el grafeno se viene incorporando a matrices poliméricas, aunque es esencial disponer de una determinada organización morfológica, control estricto de la interfase, dispersión adecuada y fácil procesabilidad para alcanzar las mejores prestaciones del nanocompuesto. Estos requisitos se pueden conseguir gracias a la versatilidad de las propiedades superficiales del grafeno y que a su vez se pueden modificar según los fines específicos. Mediante el uso de moléculas orgánicas como isocianato de fenilo ${ }^{6}$ y porfirina ${ }^{7}$ unidas a la superficie de grafeno se mejoran las dispersiones en polímeros no polares como el poliestireno (PS). En general, la dispersión de grafeno se mejora mediante su modificación estructural ${ }^{8}$.

Por lo que el propósito del presente trabajo fue de obtener nanocompuestos poliméricos con grafeno para evaluar sus propiedades mecánicas, térmicas, estructurales y morfológicas.

\section{PARTE EXPERIMENTAL}

\section{Reactivo y materiales}

Los reactivos utilizados en este trabajo fueron: dodecilsulfato de sodio (SDS), persulfato de amonio (APS), acrilato de butilo (AB), estireno (St), metacrilato de alilo (ALMA), con purezas $\geq 98,5 \%, 99,99 \%$, $\geq 99,0 \%$ y $99,0 \%$, respectivamente y suministrados por Merck Peruana. Los monómeros fueron destilados a presión reducida y almacenados a 4oC. Para la síntesis del grafeno se empleó grafito de Sigma- Aldrich. Se emplearon ácido sulfúrico, ácido clorhídrico concentrados y ácido ascórbico de Merck Peruana. Se empleó agua tridestilada, desionizada y filtrada. El nitrógeno fue grado UAP de Infra, purificado adicionalmente al pasarlo por una columna Oxiclear.

Síntesis del grafeno por el método de Hummer modificado y reducción con ácido ascórbico. Para ello se pesó $0,5 \mathrm{~g}$ de grafito y vertió en un matraz erlenmeyer de $250 \mathrm{~mL}$, colocado dentro de un baño con hielo, luego se añadió lentamente $23 \mathrm{~mL}$ de ácido sulfúrico concentrado, manteniendo a una temperatura menor de $20^{\circ} \mathrm{C}$ y en agitación por 4 hrs. Después, se agregó lentamente $3 \mathrm{~g}$ de $\mathrm{KMnO}_{4}$ y se mantuvo en agitación durante $1 \mathrm{hr}$. y en baño con hielo para evitar explosión. Luego se elevó la temperatura hasta $35^{\circ} \mathrm{C}$, retirándolo del baño con hielo, para que pueda oxidar el $\mathrm{KMnO}_{4}$, y se mantuvo en agitación por $1 \mathrm{hr}$.

Luego se adiciona $45 \mathrm{ml}$ de agua destilada y se calentó hasta $95^{\circ} \mathrm{C}$; manteniéndolo en agitación durante 2 hrs., evitando que ebulla durante la reacción. Después se calentó y agregó $100 \mathrm{ml}$ de agua destilada y se mantuvo en agitación durante 1 hr. Finalmente, se adicionó 10 
$\mathrm{ml}$ de solución de peróxido de hidrógeno al 30\%, y se agitó durante $1 \mathrm{hr}$. Una vez formado el óxido de grafeno, se procedió a lavarlo con solución de $\mathrm{HCl}$ al $5 \%$ y luego con agua destilada hasta que el pH del líquido del filtrado sea de 7.

El óxido de grafeno separado se vertió en un vaso de precipitado de $250 \mathrm{ml}$ y se agregó $200 \mathrm{ml}$ de una solución de ácido ascórbico al 5\% para lograr la reducción y se mantuvo en agitación durante 2 hrs. Para la etapa de reducción se empleó el ácido ascórbico, ya que no es tóxico en comparación con la hidracina que es empleada de manera convencional. En esta etapa puede crearse defectos topológicos y vacantes producidas durante la eliminación de los grupos funcionales ${ }^{9}$. Luego se procedió a filtrarlo y lavarlo con agua destilada hasta lograr un $\mathrm{pH}$ de 7 en el líquido de filtrado (figura 1)

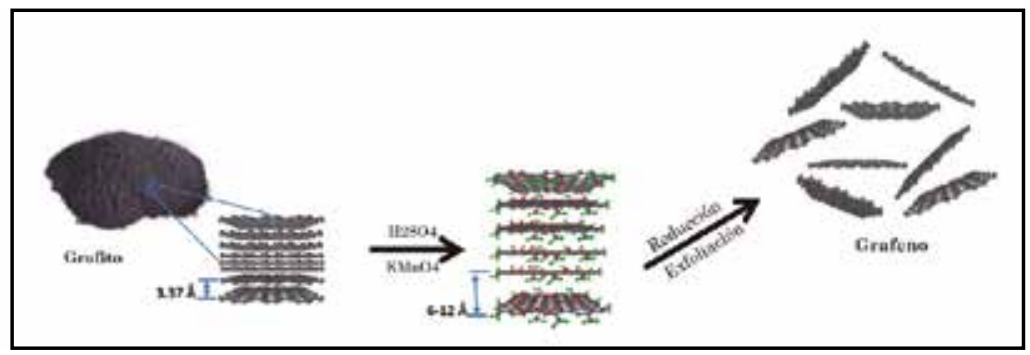

Figura 1. Síntesis de grafeno por el método de Hummer modificado

\section{Síntesis del nanocompuesto polimérico reforzado con grafeno mediante polimerización heterogénea en semicontinuo (polimerización in situ).}

Se vierte en un balón de tres bocas de $500 \mathrm{ml} \mathrm{0,2} \mathrm{g}$ de grafeno (ver tabla 1), 2 g SDS y una cantidad de 177,4 g de agua y es agitado con ultrasonido durante 3 horas en ambiente inerte, mediante la desgasificación con nitrógeno y mantenido a $60^{\circ} \mathrm{C}$ durante $1 \mathrm{hr}$. Luego se añade $0,2 \mathrm{~g}$ de persulfato de amonio (APS). Luego de 5 minutos se da inicio a la dosificación de 20 $\mathrm{g}$ de una mezcla de monómeros compuestos por estireno y acrilato de butilo en una relación másica de 40 y 60, respectivamente, mezclado con 0,2 g de metacrilato de alilo (agente entrecruzante). Para la dosificación se empleó un flujo de $0,5 \mathrm{~mL} / \mathrm{min}$ usando una bomba de jeringa "NE-300 Just Infusion".

Durante la polimerización se mantuvo la agitación por ultrasonido y el suministro de nitrógeno para mantener un ambiente inerte. Una vez terminada la dosificación de monómeros se le dio dos horas más para completar la reacción (figura 2). Al finalizar la reacción se extrajo dos muestras de $1,5 \mathrm{~mL}$ para determinar la conversión por el método gravimétrico y de $1 \mathrm{~mL}$ para determinar tamaño de partícula mediante microscopía electrónica de transmisión (TEM) y dispersión cuasielástica de luz (DLS). Los viales en los que se vierten las muestras contienen aproximadamente $0,01 \mathrm{~g}$ de una solución de hidroquinona al $0,4 \% \mathrm{p}$. 
Se prepararon látexes de nanocompuestos poliméricos con grafeno a diferentes concentraciones de grafeno mostradas en la tabla 1

Tabla 1. Muestras con diferentes contenidos de grafeno $(\% \mathrm{~m} / \mathrm{m})$

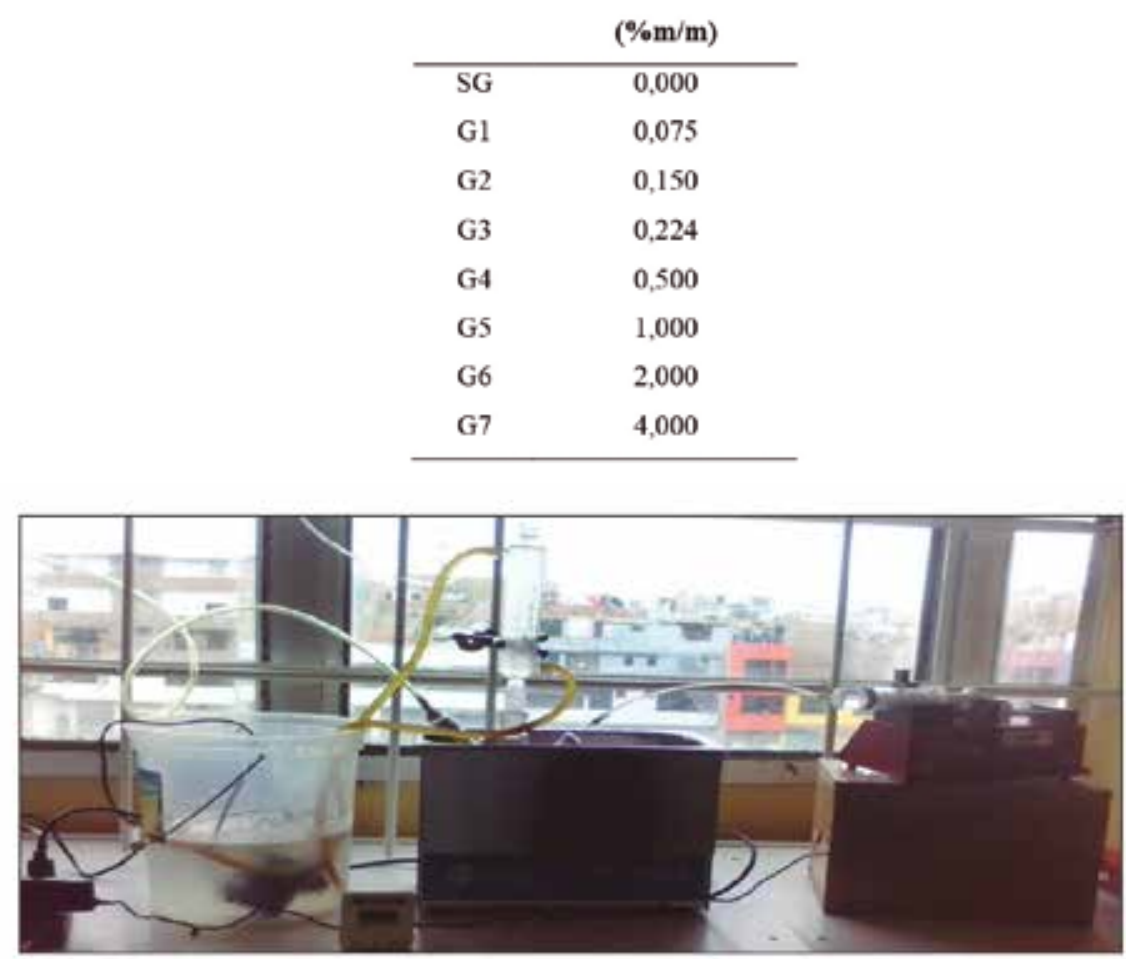

Figura 2. Sistema de obtención del nanocompuesto polimérico (polimerización in situ).

\section{Preparación de películas poliméricas a partir del látex de nanocompuesto polímero- grafeno.}

Los látex obtenidos fueron depositados en placas de vidrio y secados en una estufa a una temperatura de secado de $75^{\circ} \mathrm{C}$. Se obtuvieron películas poliméricas de 150 micras aproximadamente.

\section{Caracterización}

Para el análisis mediante espectroscopia de infrarrojo (FTIR) se empleó un equipo Thermoscinetific modelo Nicolet 8700, haciendo 16 barridos de $4000-650 \mathrm{~cm}-1$ y $4 \mathrm{~cm}-1$ de resolución en modo de ATR observando tres zonas aleatorias de la superficie de la muestra.

El análisis termogravimétrico (TGA) para evaluar la estabilidad térmica y la temperatura de descomposición del nanocompuesto polimérico se empleó analizador termogravimétrico TA 
Instruments modelo Q500. Las muestras se analizaron desde $0{ }^{\circ} \mathrm{C}$ a $800{ }^{\circ} \mathrm{C}$ a una velocidad de calentamiento de $10{ }^{\circ} \mathrm{C} / \mathrm{min}$ bajo atmósfera inerte de gas nitrógeno. El equipo fue previamente calibrado con níquel y se corrieron estándares de oxalato de calcio.

Para la determinación de la temperatura de transición vítrea se empleó un Calorímetro Diferencial de Barrido TA Instruments modelo Q200. Para el análisis se pesaron $10 \mathrm{mg}$ de muestra en charolas de aluminio, se sellaron y se colocaron en el equipo. Las condiciones de análisis se establecieron de $-70{ }^{\circ} \mathrm{C}$ a $150{ }^{\circ} \mathrm{C}$ con una velocidad de calentamiento de $10{ }^{\circ} \mathrm{C} /$ min bajo un flujo de gas nitrógeno de $50 \mathrm{~mL} / \mathrm{min}$ para mantener una atmósfera inerte.

Para la medición del diámetro de partícula se utilizó un dispersor de luz NanoBrook 90 Plus Particle Size Analyzer.

Para las pruebas mecánicas se empleó una máquina universal Shimadzu AGS-X con celda de carga de $100 \mathrm{~N}$, siguiendo la norma ASTM D-882. Los especímenes se cortaron en dimensiones rectangulares de 30 x $10 \mathrm{~mm}$ y espesor de la película original (150 micras). La velocidad de separación de las mordazas fue $300 \mathrm{~mm} / \mathrm{min}$.

\section{RESULTADOS Y DISCUSIÓN}

\section{Conversión final en las polimerizaciones}

Las conversiones al final de la polimerización, en todos los casos, fueron mayores del $80 \%$, se observó una disminución ligera de la conversión al incrementar el contenido de grafeno en el nanocompuesto, y esto es debido a que al aumentar la cantidad de grafeno retarda la movilidad de las cadenas poliméricas, haciendo que estas difícilmente reaccionen entre sí (tabla 2).

Se tiene reportado que al polimerizar in situ el ácido acrílico y acrilamida con el óxido de grafeno, usando persulfato de amonio como iniciador, se genera polímero con grafeno injertado el cual se forma por reacción de acoplamiento de radicales y exhiben una alta calidad de dispersión en agua ${ }^{10}$.

Tabla 2. Conversión final de los látex de nanocompuestos poliméricos.

\begin{tabular}{cc}
\hline Muestras & $\begin{array}{c}\text { Conversión final } \\
\text { (\%) }\end{array}$ \\
\hline SG & 94,0 \\
G1 & 93,5 \\
G2 & 90,7 \\
G3 & 88,8 \\
G4 & 88,1 \\
G5 & 87,5 \\
G6 & 87,1 \\
G7 & 87,2 \\
\hline
\end{tabular}




\section{Diámetro de partícula (Dz)}

El diámetro promedio de partícula $(\mathrm{Dz})$ es mostrado en la tabla 3, en la cual se muestra un incremento del diámetro de partícula conforme incrementa la cantidad de grafeno.

Tabla 3. Diámetros de partículas de los nanocompuestos poliméricos con grafeno.

\begin{tabular}{ccc}
\hline Muestra & $\begin{array}{c}\text { Grafeno } \\
(\mathbf{\%} \mathbf{m} / \mathbf{m})\end{array}$ & $\begin{array}{c}\text { Diámetro promedio de } \\
\text { partícula (Dz) } \\
(\mathbf{n m})\end{array}$ \\
\hline SG & 0,000 & 48,5 \\
G1 & 0,075 & 53,3 \\
G2 & 0,150 & 46,2 \\
G3 & 0,224 & 50,5 \\
G4 & 0,500 & 69,8 \\
G5 & 1,000 & 70,5 \\
G6 & 2,000 & 86,8 \\
G7 & 4,000 & 92,0 \\
\hline
\end{tabular}

\section{Análisis espectroscópico ( FTIR)}

En la figura 3 se muestra el espectro de infrarrojo de las nanoláminas de grafeno, sintetizados mediante el método de Hummer modificado y reducidos con ácido ascórbico, en la cual se muestran las bandas características de su estructura, una banda alrededor de $1600 \mathrm{~cm}^{-1}$, la cual corresponde a la vibración de estiramiento del enlace $\mathrm{C}=\mathrm{C}$, otra banda debido al óxido de grafito alrededor de $1100 \mathrm{~cm}^{-1}$ debido a la vibración del grupo éter, y también las bandas asociadas a la vibración del estiramiento y de flexión del enlace O-H alrededor de 3000-3500 $\mathrm{cm}^{-1}$ y a $1400 \mathrm{~cm}^{-1}$, respectivamente. Asimismo, se observan dos bandas a 1260 y $800 \mathrm{~cm}^{-1}$ debido a las vibraciones del grupo epoxi.

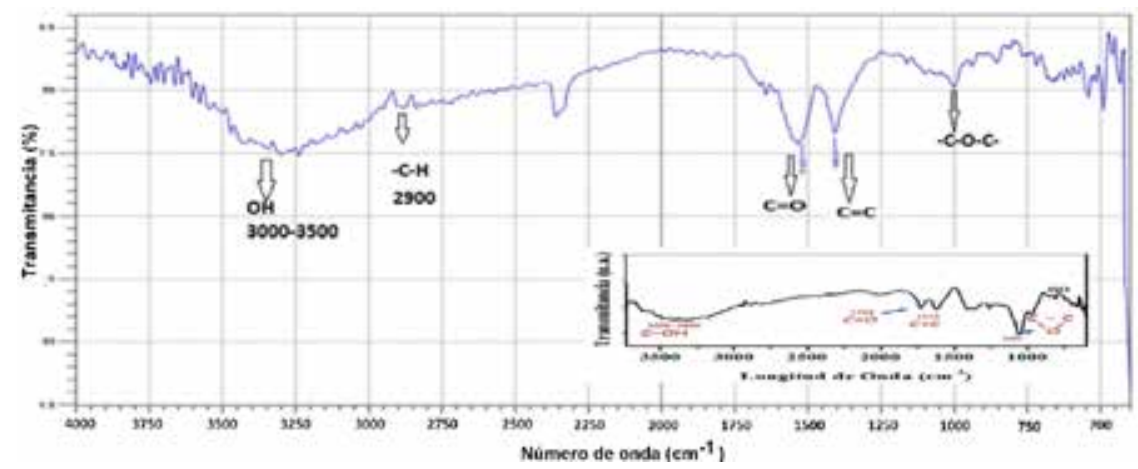

Figura 3. Espectro de infrarrojo del grafeno obtenido por el método de Hummer modificado. 
En la figura 4 se muestra el espectro de infrarrojo para el copolímero de estireno y acrilato de butilo sintetizado por polimerización heterogénea en semicontinuo, la cual se caracteriza por presentar las bandas del poliacrilato de butilo que corresponden a las bandas de estiramiento de los enlaces C-H localizadas entre los $2960-2850 \mathrm{~cm}^{-1}$, y son de intensidad media, así también presentan las bandas de flexión de C-H en 1480- $1440 \mathrm{~cm}^{-1}$. La banda intensa alrededor de la región 1780- $1640 \mathrm{~cm}^{-1}$ se debe a la presencia de los grupos carbonilo $(\mathrm{C}=\mathrm{O})$. Mientras que la presencia del poliestireno se asocia a la banda de estiramiento de $\mathrm{C}-\mathrm{H}$ aromáticos arriba de los $3000 \mathrm{~cm}^{-1}$. También se muestran los grupos metilenos del poliestireno por las bandas de estiramiento de los enlaces C-H entre los $2960-2850 \mathrm{~cm}^{-1}$, y además, presentan bandas de flexión $\mathrm{C}-\mathrm{H}$ en 1480- $1440 \mathrm{~cm}^{-1} \mathrm{y}$ finalmente las bandas de absorciones entre $1600 \mathrm{y}$ $1500 \mathrm{~cm}^{-1}$ se debe a la vibración del enlace $\mathrm{C}=\mathrm{C}$ aromático. Mientras que el metacrilato de alilo presenta los mismos grupos característicos que el poliacrilato de butilo, por lo que la detección de los metileno $\mathrm{CH}_{2}$ y C-O para el ALMA corresponden a las bandas 2960- 2850 $\mathrm{cm}^{-1}$ y para el el PAB 1300-1100 $\mathrm{cm}^{-1}$, lo que hace que las bandas sean un poco anchas e intensas.

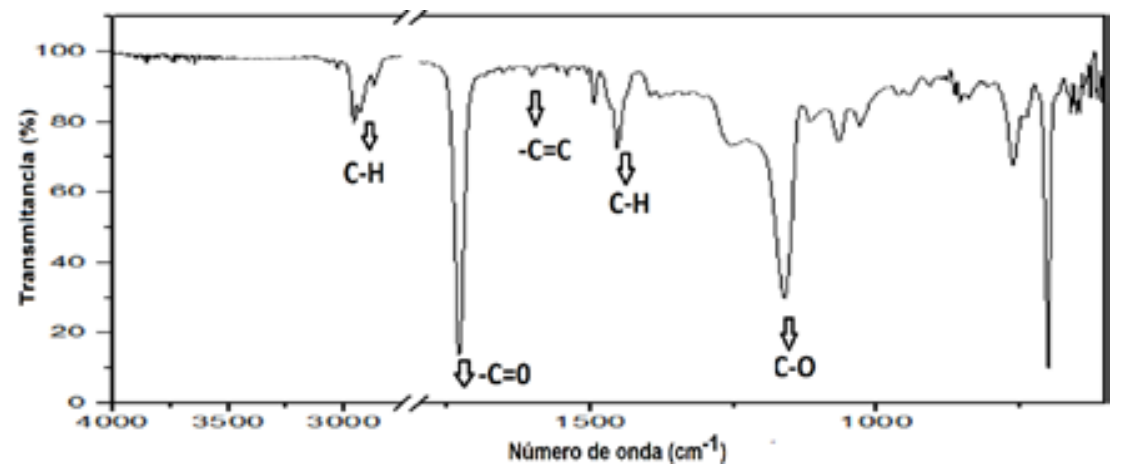

Figura 4. Espectro de infrarrojo del copolímero de estireno y acrilato de butilo.

La figura 5 muestra el espectro de infrarrojo para nanocompuestos poliméricos con una concentración de $0,224 \%$ de grafeno y se aprecia en todos que la naturaleza estructural del copolímero de estireno y acrilato de butilo se mantienen.

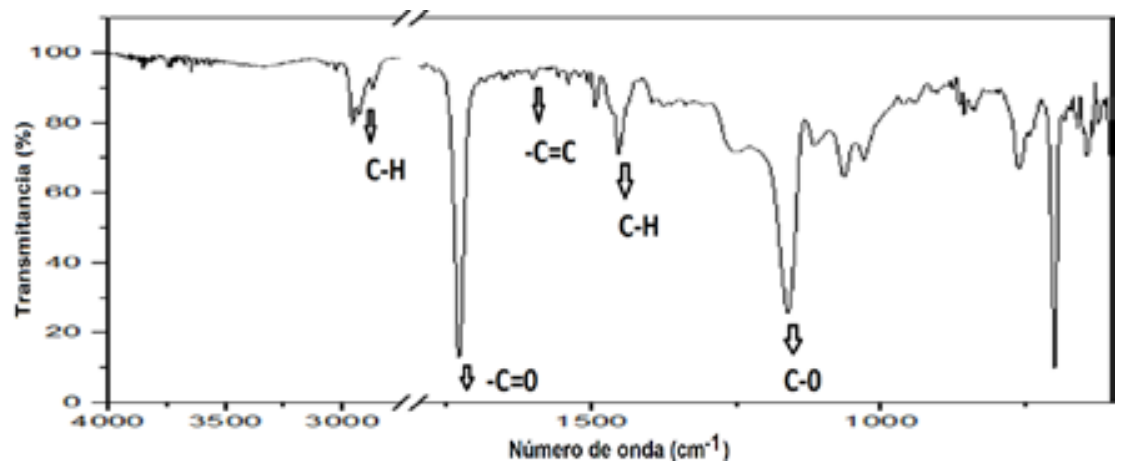

Figura 5. Espectro de infrarrojo del nanocompuesto polimérico con grafeno $0,224 \%$ (G3) 


\section{Análisis termogravimétrico (TGA)}

En este análisis se registra continuamente la masa de la muestra, colocada en atmósfera controlada, en función de la temperatura o del tiempo al ir incrementando la temperatura de la muestra. Mediante las curvas de pérdida de peso con la temperatura mostrada en la figura 6 , se determina la temperatura de degradación, o la temperatura a la cual se desorben los distintos grupos funcionales.

En la figura 6 se aprecia la pérdida de masa producida alrededor de la temperatura de $227^{\circ} \mathrm{C}$, se debe a la descomposición de los grupos funcionales oxigenados más termolábiles como son los ácidos carboxílicos entre otros ubicados en el plano basal del grafeno ${ }^{11}$.

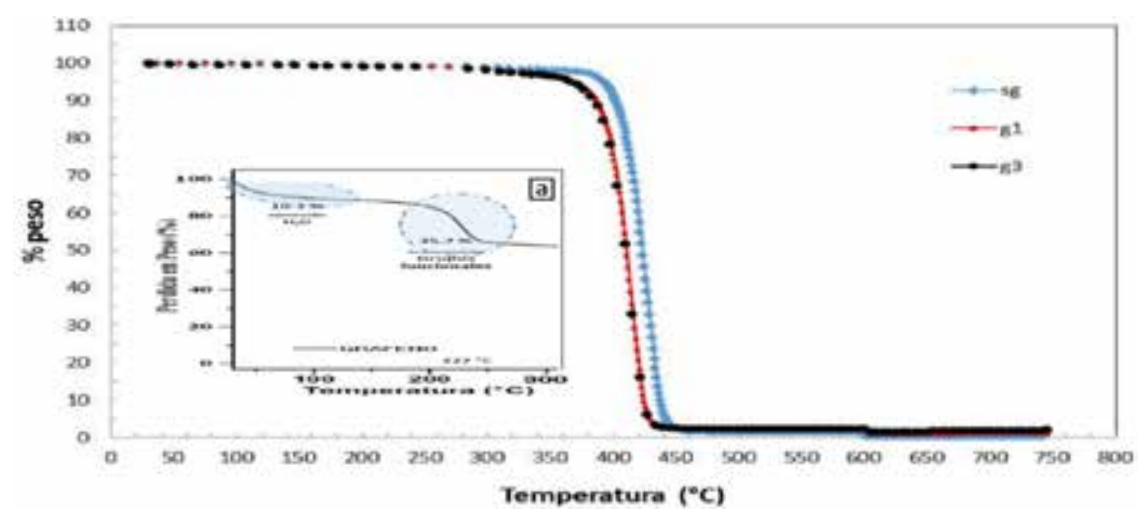

Figura 6. Comparación del análisis termogravimétrico del grafeno, copolímero y nanocompuestos poliméricos.

De acuerdo a los resultados mostrados en la tabla 4, los nanocompuestos poliméricos presentaron una disminución de la temperatura de degradación, y esto se debe a la degradación del polímero con el grafeno, siendo la temperatura de degradación del copolímero de estireno y acrilato de butilo de $425,89^{\circ} \mathrm{C}$. Se atribuye, además, que dicha disminución de la temperatura de descomposición se debe a la movilidad restringida de la cadena de copolímero cerca de la superficie del grafeno ${ }^{12}$.

Tabla 4. Temperatura de degradación del copolimero y nanocompuesto polimérico reforzado con grafeno.

\begin{tabular}{cc}
\hline Muestra & $\begin{array}{c}\text { Temperatura de degradación } \\
\left({ }^{\circ} \mathrm{C}\right)\end{array}$ \\
\hline SG & 425,89 \\
G1 & 413,21 \\
G2 & 412,15 \\
G3 & 411,09 \\
\hline
\end{tabular}




\section{Análisis por Calorimetría Diferencial de Barrido (DSC)}

En los materiales poliméricos, las transiciones térmicas más importantes en DSC son la temperatura de transición vítrea $(\mathrm{Tg})$ y la temperatura de fusión $(\mathrm{Tm})$. En los polímeros amorfos la Tg es la única transición que se registra y aparece como un cambio abrupto de la línea base. Por otra parte, en los polímeros muy cristalinos la $\mathrm{Tg}$ es difícil de observar debido a la baja proporción de la parte amorfa en el polímero ${ }^{13}$.

El incremento de la Tg en el copolímero con grafeno sintetizado por el método de Hummer modificado se atribuye a que la morfología y la funcionalización del grafeno con el polímero mejoran la interacción (tabla 5)

Tabla 5. Temperaturas de transición vítrea (Tg's) de los nanocompuestos poliméricos.

\begin{tabular}{ll}
\hline Muestra & $\begin{array}{l}\text { Tg } \\
\left({ }^{\circ} \mathbf{C}\right)\end{array}$ \\
\hline SG & 3,049 \\
G1 & 4,309 \\
G2 & 4,513 \\
G3 & 6,372 \\
\hline
\end{tabular}

\section{Caracterización mecánica de las películas del nanocompuesto polimérico}

De acuerdo a los resultados obtenidos se encontró que al agregar pequeñas cantidades de grafeno hasta $2 \% \mathrm{~m} / \mathrm{m}$, permite mejorar las propiedades mecánicas del copolímero, aumentando el módulo elástico desde 1,15 MPa hasta 5,28 MPa mientras que disminuye su deformación porcentual a rotura desde 1062 hasta $766 \%$. Sin embargo, por encima de $2 \%$ se observó que el módulo elástico del nanocompuesto disminuye debido posiblemente a que las nanopartículas de grafeno se intercalan entre las cadenas del copolímero (efecto plastificante). Por tanto, quiere decir que pequeñas cantidades de grafeno en el nanocompuesto es posible mejorar sus propiedades mecánicas; sin embargo, si se agrega mayor cantidad también es posible que haya ocurrido una disminución en su funcionalización no covalente o se haya provocado la introducción de sitios con defecto $\mathrm{sp}^{3}$ sobre la superficie del grafeno. Por tanto, al disminuir los enlaces $\mathrm{sp}^{2}$, sus propiedades mecánicas disminuyen debido a que la hibridación $\mathrm{sp}^{2}$, le da dureza, rigidez y flexibilidad al material ${ }^{14}$.

Para la estabilidad y dispersión homogénea de nanoláminas de grafeno, dentro de la matriz polimérica, se requiere la funcionalización del polímero, ya que ello reduce no sólo la interacción cohesiva debido a su tamaño sino también incrementa la interacción interfacial entre el grafeno (agente reforzante) y la matriz, debido al gran número de grupos funcionales presentes en la cadena polimérica. En este caso, se empleó el metacrilato de alilo como agente funcionalizante que cumple la función de entrecruzar y facilitar la unión química entre los polímeros, poliestireno, poliacrilato de butilo y grafeno. 
Gran parte de la investigación de nanocompuestos con nanotubos de carbono (CNT) se ha centrado en encontrar métodos para dispersar nanotubos en polímeros. Kim et al. (2010) mostraron mediante una tabla las propiedades mecánicas obtenidas por diferentes nanocompuestos poliméricos y no reportan las propiedades mecánicas de nanocompuestos poliméricos con grafeno empleando polímeros de poliestireno (material rígido) y poliacrilato de butilo (material ahulado), siendo el más cercano nanocompuesto de poliestireno con butadieno, en la cual se encontró que con una concentración de $0,8 \%$ de grafeno en el nanocompuesto se obtuvo $10 \mathrm{MPa}$ de módulo elástico y se obtuvo un incremento de su módulo en un $390 \%{ }^{15}$.

A continuación, se muestra los resultados de las pruebas mecánicas en la tabla 6 , que corresponden a las muestras de películas poliméricas de copolímero de estireno y acrilato de butilo, así como de los nanocompuestos poliméricos con grafeno obtenido por el método de Hummer modificado. A partir de estos resultados se puede observar que se mejoraron las propiedades mecánicas con el incremento del módulo elástico en un 359\% respecto al copolimero de estireno y acrilato de butilo. Asimismo, se obtuvo un módulo de Young máximo de 5,28 MPa y un \% de deformación a la rotura de $1021 \%$ a una concentración de grafeno de $2 \% \mathrm{~m} / \mathrm{m}$.

Tabla 6. Caracterización mecánica de los nanocompuestos poliméricos empleando grafeno obtenido por el Método de Hummer modificado.

\begin{tabular}{cccccc}
\hline Muestra & $\begin{array}{c}\text { Copolímero } \\
\text { St/AB }\end{array}$ & $\begin{array}{c}\text { Grafeno } \\
(\% \mathbf{\%} / \mathbf{p})\end{array}$ & $\begin{array}{c}\text { Módulo } \\
(\mathbf{M P a})\end{array}$ & $\begin{array}{c}\text { Resistencia } \\
\mathbf{( M P a )}\end{array}$ & $\begin{array}{c}\text { Deformación } \\
\text { a rotura } \\
\mathbf{( \% )}\end{array}$ \\
\hline SG & $40 / 60$ & 0,000 & $1,15 \pm 0,16$ & $3,60 \pm 0,58$ & $1062 \pm 52$ \\
G1 & $40 / 60$ & 0,075 & $1,23 \pm 0,15$ & $4,15 \pm 0,91$ & $675 \pm 45$ \\
G2 & $40 / 60$ & 0,150 & $1,53 \pm 0,36$ & $2,40 \pm 0,58$ & $709 \pm 77$ \\
G3 & $40 / 60$ & 0,224 & $2,42 \pm 0,44$ & $1,23 \pm 0,22$ & $781 \pm 90$ \\
G4 & $40 / 60$ & 0,500 & $3,13+0,44$ & $1,73+0,63$ & $698+179$ \\
G5 & $40 / 60$ & 1,000 & $3,12+1,02$ & $1,78+0,76$ & $769+203$ \\
G6 & $40 / 60$ & 2,000 & $5,28+0,49$ & $3,22+0,59$ & $1021+33$ \\
G7 & $40 / 60$ & 4,000 & $2,73+0,89$ & $1,69+0,38$ & $766+97$ \\
\hline
\end{tabular}

\section{CONCLUSIONES}

- Se obtuvo un incremento en su módulo de Young y disminución del \% de deformación conforme se incrementó la concentración de grafeno en el nanocompuesto polimérico. Asimismo, se logró obtener nanocompuesto de copolímero de estireno y acrilato de butilo (40:60) con grafeno de diámetro promedio de partícula de $86,8 \mathrm{~nm}$, con un módulo máximo de 5,28\% y a una concentración de grafeno del $2 \%$.

- Los espectros de absorción infrarrojo muestran bandas asociadas a los grupos funcionales 
epoxy, hidroxilo y carbonilo sobre la superficie del grafeno y muestran que debido a los grupos funcionales que presenta el grafeno sintetizado por el método de Hummer mejoraron las propiedades mecánicas en los nanocompuestos, ya que permitieron la unión química, que favorecen la dispersión y su estabilidad.

\section{AGRADECIMIENTOS}

Los autores agradecen la financiación otorgada por el Vicerrectorado de Investigación de la Universidad Nacional Mayor de San Marcos y a la colaboración del Centro de Investigación de Química Aplicada (CIQA)-Saltillo-México, así como del Centro de Investigación Científica de Yucatán (CICY) - Mérida-México.

\section{REFERENCIAS BIBLIOGRÁFICAS}

1. Usuki A, Kojima Y, Kawasumi M, Okada A. Fukushima Y, Kurauchi T, Kamigaito O. Synthesis of nylon 6-clay hybrid. J Mater Res. 1993; 8: 1179- 1184.

2. Balandin AA, Ghosh S, Bao W, Calizo D, Teweldebrhan D, Miao F, et al. Superior thermal conductivity of single-layer graphene. Nano Lett. 2008; 8: 902-907.

3. Geim AK. Graphene: Status and Prospects. Science 2009; 324: 1530-1534.

4. Novoselov K, Geim A, Morozov S, Jiang D, Zhang Y, Dubonos S, et al. Electric field effect in atomically thin carbon films. Science. 2004; 306(5696): 666-669.

5. Dragoman D, Dragoman M. Giant thermoelectric effect in graphene. Appl Phys Lett. 2007; 91: 203116.

6. Stankovich S, Piner R, Chen X, Wu N, Nguyen S, Ruoff R. Stable Aqueous Dispersions of Graphitic Nanoplatelets via the Reduction of Exfoliated Graphite Oxide in the Presence of Poly(sodium 4-styrenesulfonate). J Mater Chem. 2006;16: 155-158.

7. Xu Y, Liu Z, Zhang X, Wang Y, Tian J, Huang Y, Ma Y, Zhang X, Chen Y. A graphene hybrid material covalently functionalized with porphyrin: synthesis and optical limiting property. Adv Mater. 2009; 21: 1275 -1279.

8. Niyogi S, Bekyarova E, Itkis ME, McWilliams JL, Hamon MA, Haddon RCJ. Solution properties of graphite and graphene.Am Chem Soc. 2006; 128: 7720-7721.

9. Ramanathan T, Abdala AA, Stankovich S, Dikin DA, Herrera-Alonso M, Piner RD, et al. Functionalized graphene sheets for polymer nanocomposites. Nat Nanotechnol. 2008; 3(6):327-31.

10. Shen J, Hu Y, Li C, Qin C, Shi M, Ye M. Layer-by-Layer Self-Assembly of Graphene Nanoplatelets. Lagmuir 2009; 25: 6122-6128.

11. Becerril HA, Mao J, Liu Z, Stoltenberg RM, Bao Z, Yongsheng Chen Y. Evaluation of Solution-Processed Reduced Graphene Oxide Films as Transparent Conductors ACS Nano. 2008; 2(3): 463-470.

12. Liu N, Luo F, Wu H, Liu Y, Zhang C, Chen J. One step ionic-liquid assisted electrochemical synthesis of ionic-liquid-functionalized graphene sheets directly from grapheme. Adv Funct Mater. 2008; 18: 1518 - 1525 
13. Li Y, Fan Y, Ma J. Termal, physical and chemical stability of porous polystyrene-type beads with different degrees of crosslinking. Polym Degrad Stab. 2001; 73: 163-167.

14. Lee C, Wei X, Kysar J, Hone J. Measurement of the elastic properties and intrinsic strength of monolayer graphene. Science. 2008; 321: 385-388

15. Kim H,Abdala AA, Macosko CW. Graphene/polymer nanocomposites. Macromolecules. 2010; 43(16): 6515-6530. 